\title{
Filtration of UAV Movement Parameters Based on the Received Signal Strength Measurement Sensor Networks in the Presence of Anomalous Measurements of Unknown Power at the Transmitter
}

\author{
Igor Olegovych Tovkach ${ }^{1, *}$ (D), Serhii Yakovych Zhuk ${ }^{1}$ (I)
}

1. National Technical University of Ukraine - Igor Sikorsky Kyiv Polytechnic Institute - Department of Radio Engineering Devices and Systems - Kyiv - Ukraine.

*Corresponding author: tovkach.igor@gmail.com

\begin{abstract}
Methods based on received signal strength measurements (RSS measurements) are used to determine the unmanned aerial vehicle (UAV) location using a wireless sensor network. The UAV transmitter power is usually unknown. In real conditions, it often becomes necessary to consider existence of anomalous measurement results. The reasons for the violation of the measurement process can be: the influence of interference, errors in the identification of signals during primary processing, failures of the equipment and similar. The optimum and quasi-optimal adaptive algorithms of UAV movement parameters filtration based on the RSS-measurement sensor networks in the presence of anomalous measurements at the unknown power of the transmitter are developed. These algorithms are obtained using Bayes' theorems and the Markov property of a mixed process, including a vector of target movement parameters and a discrete component characterizing the type of measurement. Analysis of developed algorithm performance was carried out by Monte Carlo method on 2D plane. The quasi-optimal adaptive filtering algorithm detects the appearance of anomalous measurements with probabilities close to unity and allows one to eliminate their influence on the accuracy of UAV movement parameters estimation and also to estimate the UAV unknown transmitter power.
\end{abstract}

Keywords: UAV; RSS; Anomalous measurements; Unknown transmitter power; Adaptive algorithm; Kalman filter.

\section{INTRODUCTION}

In recent years, small unmanned aerial vehicles (UAVs) (both mini and micro) have become a popular surveillance tool in the field of defense and security. The military also increased interest in small UAVs, which can be used to solve tactical reconnaissance, electronic warfare or to deliver small bombs (Baker 2014). In many countries, continuous research and development work is being carried out in this direction. According to IC Insights, taking into account all potential areas of use, the global sales of devices in 2025 may exceed \$ 10 billion (Business Insider Intelligence 2020; Hindle 2017).

Received: Feb. 26, 2020 | Accepted: Sep. 14, 2020

Peer Review History: Double Blind Peer Review.

Section Editor: Alison Moraes

This is an open access article distributed under the terms of the Creative Commons license. 
On the other hand, the use of UAVs has led to new potential threats to national and public security. Such UAVs can carry explosives, biological or chemical weapons to carry out terrorist acts. Devices can also be used to transport smuggling, drugs, jamming GPS signals or Wi-Fi, which will lead to interruption in communication and data transfer (Wallace and Loffi 2015; Nasr et al. 2019).

The greatest vulnerability of UAVs is due to the presence of electromagnetic radiation. The standard radio frequency UAV bands are ISM $2.4 \mathrm{GHz}$ and ISM $5.8 \mathrm{GHz}$, in which most commercial Wi-Fi, Bluetooth and internet of things (IoT) systems (i.e., ZigBee, Z-Wave, LoRa) operate. The signals in these bands are freely regulated using free access rules.

Recent advances in wireless sensor networks (WSN) are opening new possibilities in solving the problem of determining radio sources location. Wireless sensor network is a set of miniature and inexpensive devices equipped with various types of sensors, a small microcontroller and a receiver, which are connected via a Wireless Local Area Network (WLAN) and uses radio channels for data transmission. This task has a wide range of applications, such as: rescue operations, autonomous surveillance and monitoring of industrial processes and the environment (monitoring of the animal world), monitoring and control of moving objects, etc.

One of the important features of wireless sensor networks is the ability to track moving objects (targets), including UAVs (Chu and Han 2019; Zhang et al. 2018). To determine the UAV location using a wireless sensor network, methods based on measuring received signal strength (RSS measurements) are the most common. One of the localization methods used in such networks is fingerprinting. In this method, the correspondence of the current signal strength is searched for with available reference values in the calibration database (Zegeye et al. 2016). Alternative to the fingerprinting method are various mathematical algorithms based on the correspondence of the received RSS measurements to the signal propagation model. The accuracy of these methods largely depends on the accuracy of the signal propagation model (Azmi et al. 2018; Uluskan et al. 2017; Li and Huang 2016; Song and Yu 2008; Zou et al. 2016). However, today there is not even a single standard that defines the types of UAVs and their application. Therefore, the UAV transmitter power is, as a rule, unknown. This makes it difficult to use signal propagation model-based techniques to locate an unknown UAV.

When solving the problem of estimating UAV movement parameters (the coordinates of position, velocity, acceleration), it is usually assumed that the measurement results contain information about the process being evaluated with some errors. Of considerable practical interest are cases in which measurements may appear that either do not contain useful information at all or contain results with significantly larger errors. Such measurements are called abnormal (rough), as opposed to the normal measurement, formed during the usual mechanism of results formation (Kupriyanov and Sakharov 2007). The reasons for the violation of the measurement process can be: the influence of interference, errors in the identification of signals during primary processing, failures of the equipment etc. The appearance of anomalous measurements in the case blinds the observer temporarily, leading to a significant decrease in the accuracy of determining the UAV location and movement parameters (Sirota and Kirsanov 2006).

The article is devoted to the development of an adaptive estimation algorithm of UAV movement parameters based on RSS measurements sensor networks at unknown transmitter power and the presence of anomalous measurements.

\section{PROBLEM DEFINITION}

The UAV movement in the rectangular coordinate system can be described by using a stochastic dynamic system with random structure having the form (Tovkach and Zhuk 2019):

$$
\mathbf{u}_{U A V}(k)=\mathbf{F}_{U A V} \mathbf{u}_{U A V}(k-1)+\mathbf{G}_{U A V} \boldsymbol{\omega}(k),
$$

where $\mathbf{u}_{U A V}^{T}(k)=(x(k), \dot{x}(k), \ddot{x}(k), y(k), \dot{y}(k), \ddot{y}(k), z(k), \dot{z}(k), \ddot{z}(k))$ is the state vector including parameters of UAV movement along coordinates of the rectangular coordinate system; $\mathbf{F}_{U A V}, \mathbf{G}_{U A V}$ are known matrices: 


$$
\begin{aligned}
& \mathbf{F}_{U A V}=\left[\begin{array}{ccc}
\mathbf{F}^{b} & 0 & 0 \\
0 & \mathbf{F}^{b} & 0 \\
0 & 0 & \mathbf{F}^{b}
\end{array}\right], \\
& \mathbf{G}_{U A V}=\left[\begin{array}{ccc}
\mathbf{G}^{b} & 0 & 0 \\
0 & \mathbf{G}^{b} & 0 \\
0 & 0 & \mathbf{G}^{b}
\end{array}\right], \\
& \mathbf{F}^{b}=\left[\begin{array}{ccc}
1 & T & \frac{T^{2}}{2} \\
0 & 1 & T \\
0 & 0 & 1
\end{array}\right], \\
& \mathbf{G}^{b}=\left[\begin{array}{c}
\frac{a \cdot T^{3}}{6} \\
\frac{a \cdot T^{2}}{2} \\
a \cdot T
\end{array}\right],
\end{aligned}
$$

$a$ is the root mean square (RMS) of random fluctuations of the rate of change of the UAV acceleration; $T$ is the period of receipt of measurements from network sensors; $\omega(k)^{T}=\left(\omega_{x}(k), \omega_{y}(k), \omega_{z}(k)\right)$ is the uncorrelated sequence of Gaussian vectors with zero expected value and identity correlation matrix $Q_{\omega}=I ; k$ - is a discrete time instant.

Wireless sensor network has $S$ sensors. For determination of UAV location on the 2D plane, the wireless sensor network has to consist of at least three sensors. The use of the RSS method must take into account the dependence of the received signal strength on the distance between the sensor and UAV. Wide range of applications use the model of signal forward propagation, which takes into account only its attenuation (Tovkach and Zhuk 2017a). In this case, the observation/measurement equation describing the measurement of UAV coordinates has the form:

$$
p^{s}(k)=h^{s}\left(\mathbf{u}_{U A V}(k)\right)+a_{j_{s}}(k) \mathrm{v}^{s}(k), s=\overline{1, S}
$$

where $p^{s}(k)$ are the measured UAV signal strength by s-th WSN sensors, $s=\overline{1, S} ; a_{j_{s}}(k) v^{s}(k)$ are the errors of UAV signal strength measurement by s-th WSN sensors; $v^{s}(k)$ are the uncorrelated sequence of Gaussian values with zero expected value and dispersion $\sigma^{2}$, which are independent.

Switching variables $a_{j_{s}}(k), j_{s}=\overline{1,2}, s=\overline{1, S}$ determine the type of measurement error. The first type of measurement error is normal $a_{1_{s}}(k)=1, s=1, S$, the second is abnormal $a_{2 s}(k)=\gamma, s=\overline{0, S}$, where $\gamma$ determines how many times the RMS of normal measurement error $\sigma$ increases. Switching variables are independent and take values with probabilities $q_{j_{s}}, j_{s}=\overline{1,2}, s=\overline{1, S}$.

Nonlinear function $h^{s}\left(\mathbf{u}_{U A V}(k)\right)$ in Eq. 6 is described by the expression: 


$$
h^{s}\left(\mathbf{u}_{U A V}(k)\right)=p^{0}-10 \cdot \alpha \times \lg \left(\frac{\sqrt{\left(x(k)-x^{s}\right)^{2}+\left(y(k)-y^{s}\right)^{2}+\left(z(k)-z^{s}\right)^{2}}}{r^{0}}\right)
$$

where $x(k), y(k), z(k)$ are UAV position coordinates; $x^{s}, y^{s}, z^{s}$ are coordinates of the s-th WSN sensors position, $s=\overline{1, S}$; $a$ is the signal attenuation coefficient (close to 2); $p^{0}$ is the signal strength at a radial distance $r^{0}$ to the sensor, which is an unknown parameter (Tomic et al. 2015). At unknown transmitter power, the parameter $p^{0}$ is unknown.

\section{DEVELOPMENT OF THE ADAPTIVE ALGORITHMS}

\section{Extending of the model state vector}

For the decision of the formulated task, the bayesian method of the adaptive filtering is applicable (Aoki 1971), whose main idea is to include unknown parameters in the state vector of the process. Following this method, the state vector of the process subject to filtering in the considered task can be extended to the form:

$$
\mathbf{u}^{T}(k)=\left[\mathbf{u}_{U A V}^{T}(k), p_{0}(k)\right]
$$

It includes a vector $\mathbf{u}_{U A V}(k)$ and UAV transmitter power $p_{0}(k)$ (Tovkach et al. 2018). In this case, the model of the dynamical system (1) is described by the expression:

$$
\mathbf{u}(k)=\mathbf{F u}(k-1)+\mathbf{G} \mathbf{\omega}(k)
$$

in which the extended matrices $\mathbf{F}$ and $\mathbf{G}$ are defined as:

$$
\mathbf{F}=\left[\begin{array}{cc}
\mathbf{F}_{U A V} & 0 \\
0 & 1
\end{array}\right] ; \quad \mathbf{G}=\left[\begin{array}{c}
\mathbf{G}_{U A V} \\
0
\end{array}\right]
$$

The equation of the RSS measurements eq. 6 does not change and the state vector $\mathbf{u}^{t r}(k)$ is replaced by the extended state vector $\mathbf{u}(k)$.

Equation 6 described the observation of a single sensor. Combine the switching variables into a vector $\Gamma(k)=\left(a_{j_{1}}(k), . ., a_{j_{S}}(k)\right)$. Also combine Eq. 6 for all sensors into a single equation:

$$
\mathbf{u}_{p}(k)=\mathbf{h}(\mathbf{u}(k))+\mathbf{v}(\Gamma(k))
$$

where $\mathbf{u}_{p}(k)=\left[p^{1}(k), \ldots, p^{S}(k)\right]^{T}$ is joint measurement vector; $\mathbf{h}(\mathbf{u}(k))=\left[h^{1}(\mathbf{u}(k)), \ldots, h^{S}(\mathbf{u}(k))\right]^{T}$ is joint vector-valued measurement function; $\mathbf{v}(\Gamma(k))=\left[a_{j_{1}}(k) v^{1}(k), \ldots, a_{j_{s}}(k) v^{S}(k)\right]^{T}$ is joint vector of measurement errors with a zero mean vector and covariance matrix $\mathbf{R}_{j_{1}, \ldots, j_{S}}(k)=\operatorname{diag}\left(a_{j_{1}}^{2}(k) \sigma^{2}, \ldots, a_{j_{s}}^{2}(k) \sigma^{2}\right)$.

\section{A posteriori probability density function of a mixed Markov process}

In Zhuk (1989), it was shown that an extended process involving a continuous component $\mathbf{u}(k)$ and discrete components $a_{j_{s}}(k), s=\overline{1, S}$, is a mixed Markov process in discrete time. The a posteriori probability density function (p.d.f.) of a mixed Markov process is described by the expression $w(\mathbf{u}(k), \Gamma(k))=P\left(\mathbf{u}(k), \Gamma(k) \mid \mathbf{U}_{p}(k)\right)$, where $\mathbf{U}_{p}(k)=\mathbf{u}_{p}(1), \ldots, \mathbf{u}_{p}(k)$. Based on the Markov property of the mixed process, Bayes' theorem and following the procedure given in Zhuk et al. (2019) p.d.f. $w(\mathbf{u}(k), \Gamma(k))$ is recurrently calculated by the equation: 


$$
\begin{aligned}
w(\mathbf{u}(k), & \Gamma(k))=P\left(\mathbf{u}_{p}(k) \mid \mathbf{u}(k), \Gamma(k)\right) \times \prod_{s=1}^{S} q_{j_{S}} \sum_{i_{1}=1}^{2} \ldots \sum_{i_{S}=1}^{2} \int_{-\infty}^{\infty} \Pi(\mathbf{u}(k) \mid \mathbf{u}(k-1)) \times \\
& \times w\left(\mathbf{u}(k-1), a_{i_{1}}(k-1), . ., a_{i_{S}}(k-1)\right) d \mathbf{u}(k-1) / P\left(\mathbf{u}_{p}(k) \mid \mathbf{U}_{p}(k-1)\right),
\end{aligned}
$$

where $\Pi(\mathbf{u}(k) \mid \mathbf{u}(k-1))=N\left(\mathbf{F u}(k-1), \mathbf{G G}^{T}\right)$ is the conditional p.d.f. defined by the eq. (5) $\left[N\left(^{*}, *\right)-\right.$ the notation for the Gaussian probability density with the corresponding mathematical expectation and correlation matrix];

$P\left(\mathbf{u}_{p}(k) \mid \mathbf{u}(k), \Gamma(k)\right)=\prod_{s=1}^{S} P\left(p^{s}(k) \mid \mathbf{u}(k), a_{j_{s}}(k)\right)$ is one-step likelihood function; $P\left(p^{s}(k) \mid \mathbf{u}(k), a_{j_{s}}(k)\right)=N\left(h^{s}(\mathbf{u}(k)), a_{j_{s}}^{2}(k) \sigma^{2}\right)$ - conditional p.d.f. defined based on the Eq. 6 and 7; $P\left(\mathbf{u}_{p}(k) \mid \mathbf{U}_{p}(k-1)\right)$ is the conditional p.d.f., executing role of a normalizing multiplier. Equation 11 describes an algorithm for nonlinear cofiltering of the state vector $\mathbf{u}(k)$ and discrete components $a_{j_{s}}(k), s=\overline{1, S}$.

Using the probability multiplication theorem, Eq. 11 can be represented as:

$$
\begin{gathered}
w_{j_{1} \ldots j_{S}}^{*}(k)=\prod_{s=0}^{S} q_{j_{s}} \\
w^{*}(\mathbf{u}(k))=\int_{-\infty}^{\infty} \Pi(\mathbf{u}(k) \mid \mathbf{u}(k-1)) w(\mathbf{u}(k-1)) d \mathbf{u}(k-1) ; \\
w_{j_{1} \ldots j_{S}}(\mathbf{u}(k))=P\left(\mathbf{u}_{p}(k) \mid \mathbf{u}(k), \Gamma(k)\right) w^{*}(\mathbf{u}(k)) / P\left(\mathbf{u}_{p}(k) \mid \Gamma(k), \mathbf{U}_{p}(k-1)\right) ; \\
w_{j_{1} \ldots j_{S}}(k)=P\left(\mathbf{u}_{p}(k) \mid \Gamma(k), \mathbf{U}_{p}(k-1)\right) \times w_{j_{1} \ldots j_{S}}^{*}(k) / P\left(\mathbf{u}_{p}(k) \mid \mathbf{U}_{p}(k-1)\right) ; \\
w(\mathbf{u}(k))=\sum_{j_{1}=1}^{2} \ldots \sum_{j_{S}=1}^{2} w_{j_{1} \ldots j_{S}}(k) w_{j_{1} \ldots j_{S}}(\mathbf{u}(k))
\end{gathered}
$$

where $w^{*}(\mathbf{u}(k))$ is the conditional predicted p.d.f. of vector $u(k) ; w_{j_{1} \ldots j_{s}}(\mathbf{u}(k))$ is the conditional a posteriori p.d.f. of the vector $\mathbf{u}(k)$ on condition $\Gamma(k) ; w(\mathbf{u}(k))$ is unconditional a posteriori p.d.f. of the vector $\mathbf{u}(k)$, defined on $k$-th step; $w_{j_{1} \ldots j_{S}}^{*}(k), w_{j_{1} \ldots j_{S}}(k)$ are predicted and a posteriori probabilities discrete components; $P\left(\mathbf{u}_{p}(k) \mid \Gamma(k), \mathbf{U}_{p}(k-1)\right)$ is the conditional p.d.f., determined by the formula:

$$
P\left(\mathbf{u}_{p}(k) \mid \Gamma(k), \mathbf{U}_{p}(k-1)\right)=\int_{-\infty}^{\infty} P\left(\mathbf{u}_{p}(k) \mid \mathbf{u}(k), \Gamma(k)\right) w^{*}(\mathbf{u}(k)) d \mathbf{u}(k)
$$

$P\left(\mathbf{u}_{p}(k) \mid \mathbf{U}_{p}(k-1)\right)$ is determined by the formula:

$$
P\left(\mathbf{u}_{p}(k) \mid \mathbf{U}_{p}(k-1)\right)=\sum_{j_{1}=1}^{2} \ldots \sum_{j_{S}=1}^{2} P\left(\mathbf{u}_{p}(k) \mid \Gamma(k), \mathbf{U}_{p}(k-1)\right) w_{j_{1} \ldots j_{S}}^{*}(k) .
$$

The initial conditions for algorithm Eqs. 12 to 16 have the form $w(\mathbf{u}(0))=P(\mathbf{u}(0))$. By means of the Eqs. 13, 14 and 16 filtering of continuous component and Eqs. 12 and 15, filtration of discrete components is executed. An inseparable connection of filtration equations for discrete and continuous components is a specific feature of this algorithm.

The optimal device realizing algorithm Eqs. 12 to 16, is multichannel with the number of channels $2^{\text {s }}$. Each channel is coordinated with a certain combination of parameters of the switching variables $\Gamma(k)$. In this case, the unconditional a posteriori p.d.f. $w(\mathbf{u}(k))$ of 
the vector $\mathbf{u}(k)$ is determined by averaging the conditional a posteriori p.d.f. $w_{j_{1} \ldots j_{s}}(\mathbf{u}(k))$ using a posteriori probabilities $w_{j_{1} \ldots j_{S}}(k)$, determined in each channel of the optimal device.

\section{DEVELOPMENT OF A QUASI-OPTIMAL ESTIMATION ALGORITHM}

Large computational costs related to the need of integrating multidimensional p.d.f. and also the considerable number of channels in case of big $S$ are a disadvantage of the optimal algorithm. A quasi-optimal adaptive filtering algorithm can be obtained by linearizing Eq. 6 and a Gaussian approximation of the $a$ posteriori p.d.f. $w(u(k))$. At the same time, predicted p.d.f. $w^{*}(u(k))$ is also Gaussian and its expected value $u^{*}(k)$ and covariance matrix $P^{*}(k)$ are calculated by the formulas (Zhuk 1989):

$$
\begin{gathered}
\mathbf{u}^{*}(k)=\mathbf{F} \hat{\mathbf{u}}(k-1) \\
\mathbf{P}^{*}(k)=\mathbf{F} \hat{\mathbf{P}}(k-1) \mathbf{F}^{T}+\mathbf{G}_{j_{0}} \mathbf{G}_{j_{0}}^{T}
\end{gathered}
$$

where $\hat{\mathbf{u}}(k-1), \hat{\mathbf{P}}(k-1)$ are expected value and correlation matrix of a posteriori p.d.f $w(\mathbf{u}(k-1))$.

Determination of a posteriori p.d.f. $w(\mathbf{u}(k))$ (13) comes down to calculating its expected value $\hat{\mathbf{u}}(k)$ and covariance matrix $\hat{\mathbf{P}}(k)$ (Evlanov and Zhuk 1990) using notation $\tilde{p}^{s}(k)=p^{s}(k)-h^{s}\left(\hat{\mathbf{u}}^{s-1}(k)\right)$ :

$$
\begin{aligned}
& H^{s}(k)=\frac{\partial h^{s}\left(\hat{\mathbf{u}}^{s-1}(k)\right)}{\partial \mathbf{u}(k)} \cdot \frac{\partial h^{s}\left(\hat{\mathbf{u}}^{s-1}(k)\right)}{\partial \mathbf{u}(k)}=\frac{-10 \cdot \alpha}{\ln (10)} \times \\
& \times\left[\frac{\hat{x}(k-1)-x^{s}}{\left(\hat{x}(k-1)-x^{s}\right)^{2}+\left(\hat{y}(k-1)-y^{s}\right)^{2}+\left(\hat{z}(k-1)-z^{s}\right)^{2}} 00\right. \\
& \frac{\hat{y}(k-1)-y^{s}}{\left(\hat{x}(k-1)-x^{s}\right)^{2}+\left(\hat{y}(k-1)-y^{s}\right)^{2}+\left(\hat{z}(k-1)-z^{s}\right)^{2}} 00 \\
& \left.\frac{\hat{z}(k-1)-z^{s}}{\left(\hat{x}(k-1)-x^{s}\right)^{2}+\left(\hat{y}(k-1)-y^{s}\right)^{2}+\left(\hat{z}(k-1)-z^{s}\right)^{2}} \begin{array}{lll}
0 & 0 & 1
\end{array}\right]^{T} \text {. }
\end{aligned}
$$

In synthesis of the quasi-optimal algorithm, a sequential method of processing incoming data is used (Tovkach et al. 2019). The procedure is recurrent when measurements are received from the s-th sensor. In the first step, the expected value $\hat{\mathbf{u}}_{j_{s}}^{s}(k)$ and correlation matrix $\hat{\mathbf{P}}_{j_{s}}^{s}(k)$ of conditional a posteriori p.d.f. $w_{j_{s}}(\mathbf{u}(k))$ are calculated, provided that the value of the switching variable $a_{j_{s}}(k)$ is known precisely. These equations coincide with the well-known equations of the extended Kalman filter:

$$
\begin{gathered}
\mathbf{K}_{j_{s}}^{s}(k)=\hat{\mathbf{P}}^{s-1}(k) H^{s T}(k)\left(d_{j_{s}}^{s}(k)\right)^{-1} \\
\hat{\mathbf{u}}_{j_{s}}^{s}(k)=\hat{\mathbf{u}}^{s-1}(k)+\mathbf{K}_{j_{s}}^{s}(k) \tilde{p}^{s}(k) \\
\hat{\mathbf{P}}_{j_{s}}^{s}(k)=\hat{\mathbf{P}}^{s-1}(k)-\mathbf{K}^{s}(k) H^{s}(k) \hat{\mathbf{P}}^{s-1}(k)
\end{gathered}
$$

where $\mathbf{K}_{j_{s}}^{s}(k)$ - quasi-optimal filter channel gain; $d_{j_{s}}^{s}(k)$ is the dispersion of a discrepancy determined by a formula: $d_{j_{s}}^{s}(k)=H^{s}(k) \hat{\mathbf{P}}^{s-1}(k) H^{s T}(k)+a_{j_{l}}^{2}(k) \sigma^{2}$ 
At the second step, in contrast to the Kalman filter, this algorithm calculates the posterior probabilities $w_{j_{s}}(k)$ of values of the switching variable $a_{j_{s}}(k)$ by the formula:

$w_{j_{s}}(k)=P\left(p^{s}(k) \mid a_{j_{s}}(k), p^{1}(k), \ldots, p^{s-1}(k), \mathbf{U}_{p}(k-1)\right) \times p_{j_{s}} / P\left(p^{s}(k) \mid p^{1}(k), \ldots, p^{s-1}(k), \mathbf{U}_{p}(k-1)\right) ;$

$P\left(p^{s}(k) / a_{j_{s}}(k), p^{1}(k), \ldots, p^{s-1}(k), \mathbf{U}_{p}(k-1)\right)$ is the conditional p.d.f. measurements $p^{s}(k)$, determined by the formula

$$
P\left(p^{s}(k) \mid a_{j_{s}}(k), p^{1}(k), \ldots, p^{s-1}(k), \mathbf{U}_{p}(k-1)\right)=N\left(h^{s}\left(\hat{\mathbf{u}}^{s-1}(k)\right), d_{j_{s}}^{s}(k)\right)
$$

$w_{j_{s}}(k)=P\left(a_{j_{s}}(k) \mid p^{1}(k), \ldots, p^{s}(k), \mathbf{U}_{p}(k-1)\right)$ is a posteriori probability $a_{j_{s}}(k)$, derived from measurements $p^{1}(k), \ldots, p^{s}(k)$, $\mathbf{U}_{p}(k-1) ; P\left(p^{s}(k) \mid p^{1}(k), \ldots, p^{s-1}(k), \mathbf{U}_{p}(k-1)\right)$ is the conditional p.d.f. measurements $p^{s}(k)$, determined by the formula:

$P\left(p^{s}(k) \mid p^{1}(k), \ldots, p^{s-1}(k), \mathbf{U}_{p}(k-1)\right)=\sum_{j_{s}=1}^{2} q_{j_{s}} \times P\left(p^{s}(k) \mid a_{j_{s}}(k), p^{1}(k), \ldots, p^{s-1}(k), \mathbf{U}_{p}(k-1)\right)$

In contrast to the Kalman filter, at the third step, the unconditional estimate of the continuous component $\hat{\mathbf{u}}^{s}(k)$ and the correlation matrix of the estimation error $\hat{\mathbf{P}}^{s}(k)$ are calculated by the formulas

$$
\begin{gathered}
\hat{\mathbf{u}}^{s}(k)=\sum_{j_{l}=1}^{2} \hat{\mathbf{u}}_{j_{s}}^{s}(k) w_{j_{s}}(k) \\
\hat{\mathbf{P}}^{s}(k)=\sum_{j_{s}=1}^{2} \hat{\mathbf{P}}_{j_{s}}^{s}(k) w_{j_{s}}(k), s=\overline{1, S}
\end{gathered}
$$

Estimated $\hat{\mathbf{u}}^{s}(k)$ and the correlation matrix of the estimation error $\hat{\mathbf{P}}^{s}(k)$ are the mathematical expectation and the correlation matrix of the Gaussian approximation of the a posteriori p.d.f. $w(\mathbf{u}(k))$. This allows to obtain a quasi-optimal device with a number of channels equal to two.

Initial conditions for the procedure Eqs. 17 to 24 at $s=0$ have the form $\hat{\mathbf{u}}^{0}(k)=\mathbf{u}^{*}(k), \hat{\mathbf{P}}^{0}(k)=\mathbf{P}^{*}(k)$, and, respectively, resultant values in case at $s=\mathrm{S}$ have the form $\hat{\mathbf{u}}(k)=\hat{\mathbf{u}}^{S}(k), \hat{\mathbf{P}}(k)=\hat{\mathbf{P}}^{S}(k)$.

In case of implementation of the procedure Eqs. 19 to 24 on each $l$-th step, Gaussian approximation of $a$ posteriori p.d.f. $P\left(\mathbf{u}(k) \mid p^{1}(k), \ldots, p^{s}(k), \mathbf{U}_{p}(k-1)\right)$ is executed and only the moments remain $\hat{\mathbf{u}}^{s}(k), \hat{\mathbf{P}}^{s}(k), s=\overline{1, S}$.

\section{ANALYSIS OF PERFORMANCE OF THE ALGORITHM}

Analysis of algorithm performance Eqs. 17 to 24 was carried out by Monte Carlo method on 2D-plane. The wireless sensor network, represented in Fig. 1, consists of eight sensors located at the following: 
Table 1. The position sensors of the wireless sensor network.

\begin{tabular}{ccccccccc}
\hline $\mathbf{S}$ & $\mathbf{1}$ & $\mathbf{2}$ & $\mathbf{3}$ & $\mathbf{4}$ & $\mathbf{5}$ & $\mathbf{6}$ & $\mathbf{7}$ & $\mathbf{8}$ \\
\hline $\mathrm{X}$ & 0 & 70.71 & 100 & 70.71 & 0 & -70.71 & -100 & -70.71 \\
\hline $\mathrm{Y}$ & 100 & 70.71 & 0 & -70.71 & -100 & -70.71 & 0 & 70.71 \\
\hline
\end{tabular}

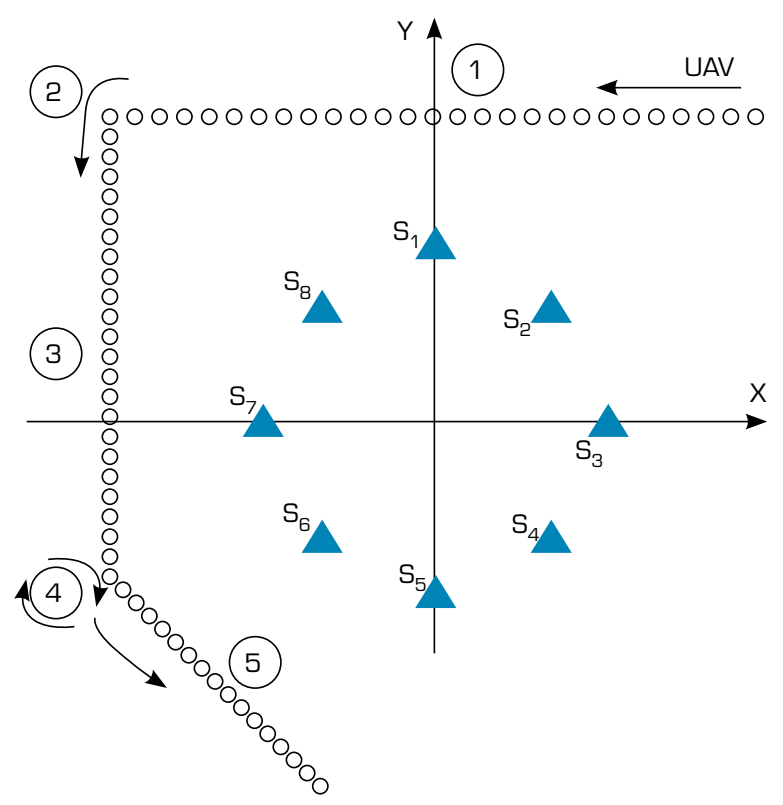

Figure 1. Configuration of the sensor network consisting of eight sensors and UAV movement trajectory.

For clarity of the algorithm, a test UAV motion trajectory (Fig. 1) was formed. It contains $k=\overline{1,130}$ steps. The trajectory consists of five sections (Tovkach and Zhuk 2017b). Abnormal measurement errors occur in the following time instants: for the first sensor $k=14,85,87$; for the second sensor $k=26,27,28$; for the sixth sensor $k=55$; for the seventh sensor $k=105$. Error RMS of measurement is $\sigma=1 \mathrm{dBm}$, the rate of receipt of measurements from network sensors $\mathrm{T}=1 \mathrm{~s}$, number of realizations is $L=100$. The simulation was conducted with $a=3 \mathrm{~m} / \mathrm{s}^{3}$. The probability of appearance of anomalous sensor measurements at any sensor is equal to $q_{2_{s}}=0.01, s=\overline{1, S}$, and parameter $\gamma=6$.

Using the Monte Carlo method means, $m_{\hat{\varepsilon}_{y}}(k), m_{\hat{\varepsilon}_{x}}(k)$ and RMS errors $\sigma_{\hat{\varepsilon}_{x}}(k), \sigma_{\hat{\varepsilon}_{y}}(k)$ of estimation UAV locations were calculated by the formulas:

$$
\begin{gathered}
m_{\hat{\varepsilon}_{x}}(k)=\frac{1}{L} \sum_{l=1}^{L} \hat{\varepsilon}_{x}^{l}(k)=\frac{1}{L} \sum_{l=1}^{L}\left(\hat{x}^{l}(k)-x(k)\right), k=\overline{1,130} \\
m_{\hat{\varepsilon}_{y}}(k)=\frac{1}{L} \sum_{l=1}^{L} \hat{\varepsilon}_{y}^{l}(k)=\frac{1}{L} \sum_{l=1}^{L}\left(\hat{y}^{l}(k)-y(k)\right), k=\overline{1,130} \\
\sigma_{\hat{\varepsilon}_{x}}(k)=\sqrt{\frac{1}{L-1} \sum_{l=1}^{L}\left(\hat{\varepsilon}_{x}^{l}(k)-m_{\hat{\varepsilon}_{x}}(k)\right)^{2}}, k=\overline{1,130} \\
\sigma_{\hat{\varepsilon}_{y}}(k)=\sqrt{\frac{1}{L-1} \sum_{l=1}^{L}\left(\hat{\varepsilon}_{y}^{l}(k)-m_{\hat{\varepsilon}_{y}}(k)\right)^{2}}, k=\overline{1,130}
\end{gathered}
$$


where $\hat{x}^{l}(k), \hat{y}^{l}(k)$ are estimations of the $X, Y$ coordinates on the $k$-th step in the $l$-th test (trajectory); $x(k), y(k)$ are true coordinates; $\hat{\varepsilon}_{x}^{l}(k), \hat{\varepsilon}_{y}^{l}(k)$ are $X, Y$ coordinates estimation errors at the $k$-th step in the $l$-th test (trajectory); $L$ is the number of tests.

Figure 2 shows RMS (curve 3) errors of estimation $\sqrt{\hat{P}_{11}(k)}, \sqrt{\hat{P}_{44}(k)}$ of the coordinates $X, Y$, obtained when algorithm Eqs. 17 to 24 is executed. Also Fig. 2 shows mean (curve 1) $m_{\hat{\varepsilon}_{y}}(k), m_{\hat{\varepsilon}_{x}}(k)$ and RMS (curve 2) $\sigma_{\hat{\varepsilon}_{x}}(k), \sigma_{\hat{\varepsilon}_{y}}(k)$ errors of estimation, obtained by statistical simulation. Theoretical and actual RMS errors estimation are close, which indicates the correct operation of algorithm.

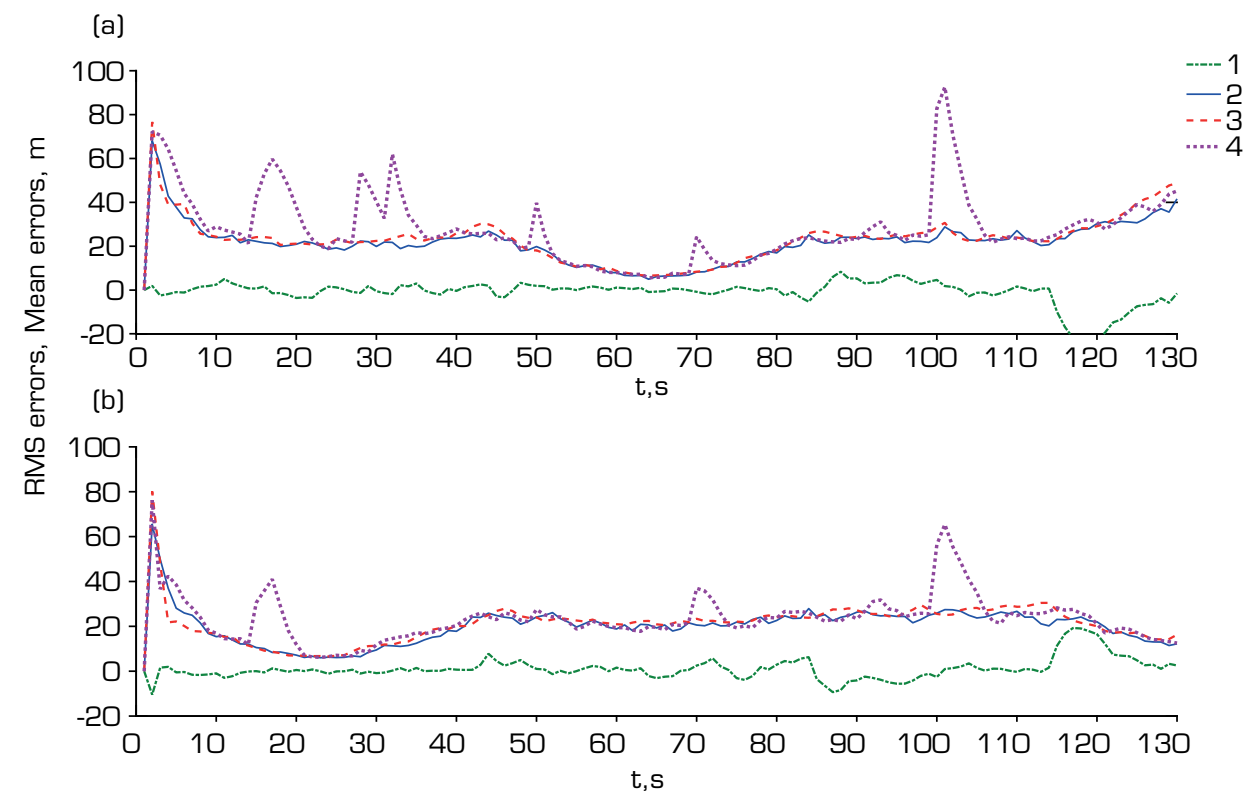

Figure 2. Characteristics of error in estimating the UAV localization while using an adaptive filter and Kalman filter. (a) Estimation errors of X-coordinate ; (b) Estimation errors of Y-coordinate.

Also, Fig. 2 shows RMS (curve 4) $\sigma_{\hat{\varepsilon}_{x}}(k), \sigma_{\hat{\varepsilon}_{y}}(k)$ errors of estimation when using the Kalman filter obtained by statistical modeling. Figure 3 shows the relationships of the probabilities of detecting anomalous errors of measurements for all sensors. The adaptive filter provides a high probability of recognition of abnormal measurements.

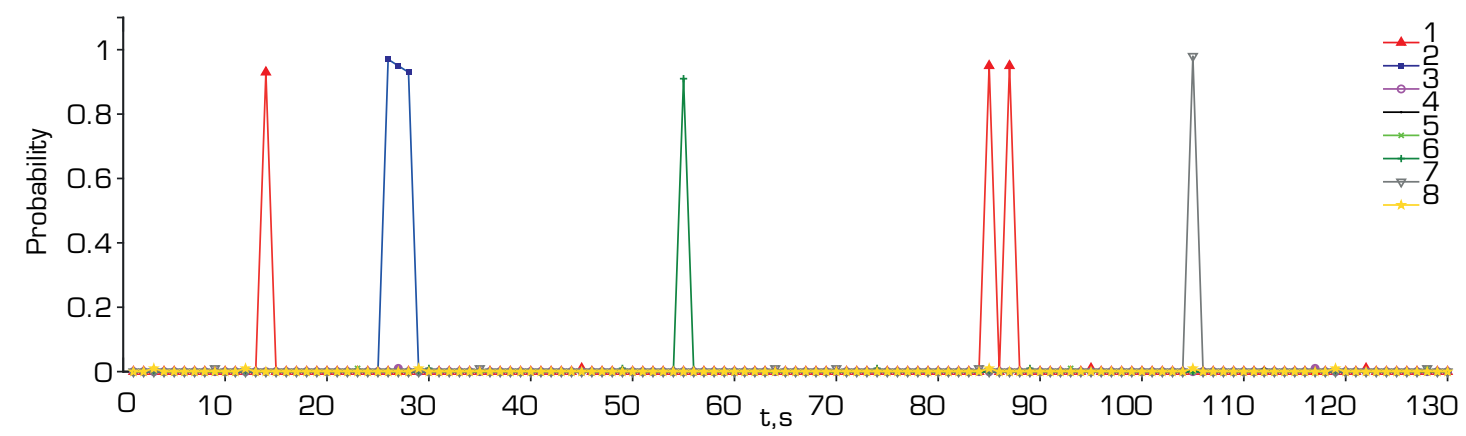

Figure 3. The probability of detecting anomalous measurement errors when using adaptive filter.

Figure 4 shows RMS (curve 3) errors of UAV transmitter power estimation and also their means (curve 1) and RMS (curve 2) using the algorithm Eqs. 17 to 24, obtained by statistical simulation. The developed filter allows reducing the RMS (curve 3) of UAV transmitter power errors from 5 to $0.3 \mathrm{dBm}$ (Tovkach et al. 2018). After completing the adaptation process, occupying 30 steps, the developed filter allows to determine the location of the UAV with the accuracy characteristics close to an algorithm, in which the known power of the transmitter is used. 


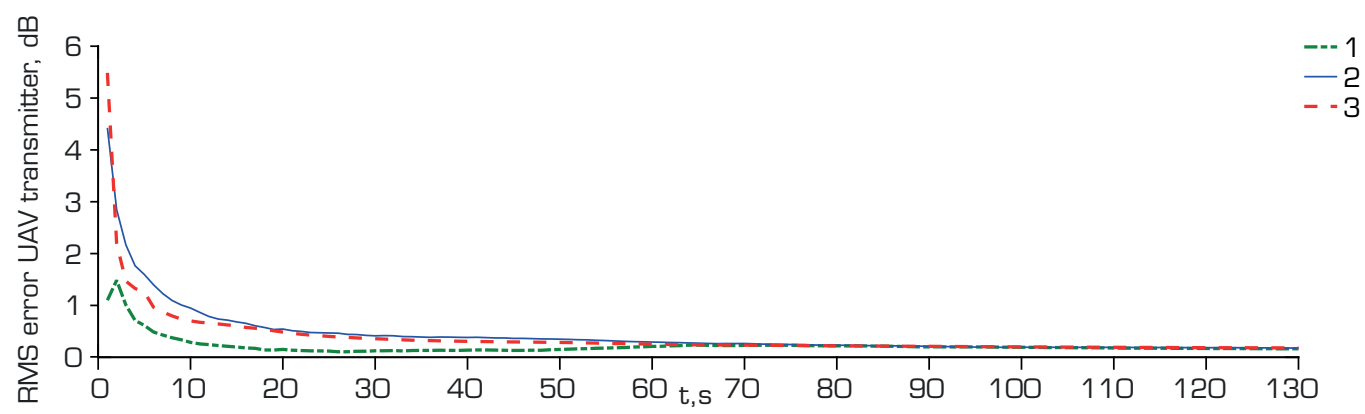

Figure 4. RMS errors of the UAV transmitter power estimation when using adaptive filter.

As follows from Fig. 2, the appearance of anomalous measurement errors leads to a significant increase in the estimation errors of the Kalman filter. The application of the developed quasi-optimal adaptive algorithm of trajectory filtering allows one to eliminate their influence on the accuracy of estimating UAV movement parameters in comparison with the Kalman filtering algorithm, as shown in Fig. 2. When abnormal measurement errors appear, the RMS error of the estimation increases by 2 - 5 times when using the Kalman filter.

\section{CONCLUSIONS}

To identify the unknown transmitter power, the method of expanding the state vector is applied, according to which the unknown parameter is included in the state vector of the filtered process.

Using the optimal adaptive filtering algorithm (Eqs. 12 to 16), the calculation of the joint a posteriori p.d.f. state vector and switching variables is performed. It is recurrent, due to the Markov property of the extended mixed process. The optimal filter is multichannel. Each channel is matched to a specific combination of switching variable parameters. The disadvantage of the optimal algorithm is the large computational costs associated with the need to integrate multidimensional p.d.f., as well as a significant number of filter channels.

The quasi-optimal adaptive filtering algorithm (Eqs. 17 to 24) was obtained by linearizing the measurement in Eq. 6 and Gaussian approximation of the a posteriori p.d.f. state vector. It performs sequential processing of incoming measurements. The number of channels of the quasi-optimal device is equal to two. The inclusion of unknown transmitter power in the state vector allows simultaneously with solving the main task of estimating UAV movement parameters, also to estimate its power, which can be useful for UAVs identification.

The quasi-optimal adaptive filtering algorithm detects the appearance of anomalous measurements with probabilities close to unity and allows one to eliminate their influence on the accuracy of UAV movement parameters estimation in comparison with the Kalman filtering algorithm. After completing the adaptation process of RMS (curve 3) of UAV, transmitter power error is $0.3 \mathrm{dBm}$.

A further area of research is the development of trajectory filtering algorithms for maneuvering UAVs with the integrated use of data from the sensor network, using the time difference of arrival (TDOA), angle of arrival (AOA) and RSS methods.

\section{AUTHOR'S CONTRIBUTION}

Conceptualization: Tovkach IO and Zhuk SY; Methodology: Tovkach IO and Zhuk SY; Investigation: Tovkach IO and Zhuk SY; Writing: Tovkach IO.and Zhuk SY.

\section{DATA AVAILABILITY STATEMENT}

All data sets were generated or analyzed in the current study. 


\section{FUNDING}

Not applicable.

\section{ACKNOWLEDGEMENTS}

Not applicable.

\section{REFERENCES}

Aoki M (1971) Optimizatsiya stokhasticheskikh sistem: Diskretnoe vremya (Optimization of Stochastic Systems: Discrete Time), Moscow: Nauka.

Azmi NA, Samsul S, Yamada Y, Yakub MFM, Ismail MIM, Dziyauddin RA (2018) A Survey of Localization using RSSI and TDoA Techniques in Wireless Sensor Network: System Architecture. Paper presented 2018 2nd International Conference on Telematics and Future Generation Networks (TAFGEN). IEEE; Kuching, Malaysia. https://doi.org/10.1109/ TAFGEN.2018.8580464

Baker B (2014) Small bombs, big effect: arming small UAVs with guided weapons. [accessed Dec 20 2020]. https://www. airforce-technology.com/features/featuresmall-bombs-big-effect-arming-small-uavs-with-guided-weapons-4467893/

Business Insider Intelligence (2020). Commercial unmanned aerial vehicle (UAV) market analysis - industry trends, forecasts and companies. [accessed Dec 20 2020]. http://www.businessinsider.com/commercial-uav-market-analysis-2017-8

Chu J, Han W (2019) Internet Communication system protocol based on wireless sensor. Radioelectron Commun Syst 62(8):422-429. https://doi.org/10.3103/S0735272719080065

Evlanov PA, Zhuk SY (1990) Integration of meters with failures. Radioelectronics and Communications Systems 33(7):49-53. https://doi. org/10.3103/S073527271990070111

Hindle P (2017) Drone Detection and Location Systems. Microwave Journal.

Kupriyanov AI, Sakharov AV (2007) Theoretical foundations of electronic warfare: Textbook. Moscow: University Book.

Li D, Huang J (2016) RSS based method for sensor localization with unknown transmit power and uncertainty in path loss exponent. Paper presented 2016 8th IEEE International Conference on Communication Software and Networks (ICCSN). IEEE; Beijing, China. https://doi.org/10.1109/ICCSN.2016.7586668

Nasr I, Chekir M, Besbes H (2019) Shipwrecked victims localization and tracking using UAVs. Paper presented 201915 th International Wireless Communications \& Mobile Computing Conference (IWCMC). IEEE; Tangier, Morocco. https://doi. org/10.1109/IWCMC.2019.8766534

Sirota AA, Kirsanov E (2006) The neural-network and statistical algorithms for estimating coordinates of a source of radio radiation in multi-position radio systems in the presence of abnormal errors of primary parameter measurement. Radioelectron Commun Syst 49(4):13-18.

Tomic S, Beko M, Dinis R (2015) RSS-based localization in wireless sensor networks using convex relaxation: noncooperative and cooperative schemes. IEEE Trans Veh Technol 64(5):2037-2050. https://doi.org/10.1109/TVT.2014.2334397 
Tovkach IO, Zhuk SY (2017a) Adaptive filtration of radio source movement parameters with complex use of sensor network data based on TDOA and RSS methods. Radioelectron Commun Syst 60(12):528-537. https://doi.org/10.3103/S0735272717120020

Tovkach IO, Zhuk SY (2017b) Adaptive filtration of parameters of the UAV movement on data from its location calculated on the basis the time difference of arrival method. Presented at: IEEE First Ukraine Conference on Electrical and Computer Engineering; Kiev, Ukraine. https://doi.org/10.1109/ukrcon.2017.8100466

Tovkach IO, Neuimin OS, Zhuk SY (2018) Filtration of parameters of the UAV movement based on the RSS-measurement at the unknown power of the transmitter. Paper presented 2018 14th International Conference on Advanced Trends in Radioelecrtronics, Telecommunications and Computer Engineering (TCSET). IEEE; Slavske, Ukraine. https://doi. org/10.1109/TCSET.2018.8336155

Tovkach IO, Zhuk SY (2019) Adaptive filtration of parameters of the UAV movement based on the TDOA-measurement sensor networks. J Aerosp Technol Manag 11:e3519. https://doi.org/10.5028/jatm.v11.1062

Tovkach IO, Zhuk SY, Reutska Y, Neuimin OS (2019) Estimation of radio source movement parameters based on TDOAand RSS-measurements of sensor network in presence of anomalous measurements. Paper presented 2019 IEEE 39th International Conference on Electronics and Nanotechnology (ELNANO). IEEE; Kyiv, Ukraine. https://doi.org/10.1109/ ELNANO.2019.8783384

Uluskan S, Gökçe M, Filik T (2017) RSS based localization of an emitter using a single mini UAV. Paper presented 2017 25th Signal Processing and Communications Applications Conference (SIU). IEEE; Antalya, Turkey. https://doi.org/10.1109/ SIU.2017.7960239

Song Y, Yu H (2008) A new hybrid TOA/RSS location tracking algorithm for wireless sensor network. Paper presented 2008 9th International Conference on Signal Processing. IEEE; Beijing, China. https://doi.org/10.1109/ICOSP.2008.4697692

Wallace RJ, Loffi JM (2015) Examining unmanned aerial system threats \& defenses: a conceptual analysis. Int J Aviat Aeronaut Aerosp 2(4):1-33. https://doi.org/10.15394/ijaaa.2015.1084

Zegeye WK, Amsalu SB, Astatke Y, Moazzami F (2016) WiFi RSS fingerprinting indoor localization for mobile devices. Paper presented 2016 IEEE 7th Annual Ubiquitous Computing, Electronics \& Mobile Communication Conference (UEMCON). IEEE; New York, New York, United States. https://doi.org/10.1109/UEMCON.2016.7777834

Zhang X, Huang J, Wang Y, Zhou Y (2018) An efficient estimator for target localization in a multistation redundancy system without matrix inversion. J Sens 2018:6362939. https://doi.org/10.1155/2018/6362939

Zhuk SY (1989) Synthesis of digital detector-meters for mixed Markovian processes. Radioelectron Commun Syst 32(11):2934. https://doi.org/10.3103/S073527271989110063

Zhuk SY, Tovkach IO, Reutska YY (2019) Adaptive filtration of radio source movement parameters based on sensor network TDOA measurements in presence of anomalous measurements. Radioelectron Commun Syst 62(2):61-71. https://doi. org/10.3103/S073527271902002X

Zou T, Lin S, Li S (2016) Blind RSSD-based indoor localization with confidence calibration and energy control. Sensors 16(6):788. https://doi.org/10.3390/s16060788 